\title{
MULTI-PERIOD AGE-DISCRIMINATED PERISHABLE INVENTORY
}

\author{
Wasfi ALRAWABDEH \\ The Hashemite University
}

\begin{abstract}
:
In this paper, an extremely short shelf-life inventory of age-discriminated stochastic demand is considered. Age discriminated demand can be found in products of high circulation and short shelf-lives such as dairy products, packaged food, pharmaceutical products and medical products of short shelf lives. Simulation based optimization is considered to find the optimal order quantity. The model employs Discrete Event Simulation along with a modified simulated annealing algorithm. To validate the model and the optimization algorithm, the classical newsvendor problem is tested first, later, different experiments are carried out for different product lifetimes. In contrast to the classical newsvendor, this problem tackles a multi-period inventory of different ages and different demand distributions. The objective is to determine the optimal order quantity to satisfy the stochastic demand of all ages such that shortages and expirations are minimized. The results showed remarkable performance and outstanding minimum levels of shortage and expiration.
\end{abstract}

Key words: probabilistic inventory, demand, age discrimination, newsvendor, supply chain

\section{INTRODUCTION}

Perishable products have a short shelf life, such as dairy products, fish, fruit, vegetables, pharmaceuticals and pesticides, which undergo a deterioration process as they get older. To have a sense of the size of investment in perishable products, it is sufficient to know that the U.S. retail industry has been estimated to raise more than $\$ 600$ billion in annual revenue, with about $55 \%$ in perishable revenue [1]. Deterioration typically leads to a drop in the market value of products and, by the end of the day, the valid amount offered for sale declines. Consequently, the loss of perishable products affects the decision on inventories in the distribution chain. Products that undergo changes in its functional integrity are said to be perishable; therefore, in order to increase revenue and minimize losses due to decay and damage, many businesses apply marketing techniques, such as pricing and advertising along with inventory management, to enhance the consumer's willingness to buy. In reality, relying on production-inventory decisions by implementing marketing approaches are especially relevant for perishable goods. Supply chain management of perishable goods is a highly challenging task with several factors that have to be taken into consideration. Such factors derive from the fact that perishable goods lose consistency and reliability even under proper conditions in the supply chain. Since living standards are growing and consumers are primarily opt- ing for high-quality fresh foods, as opposed to dried, frozen or otherwise non-perishable products, the need for appropriate strategies to control supply chains of perishable goods is significantly important.

The biggest challenge in designing management schemes for perishable inventories is the requirement of detailed commodity lifetime study. In the case, where demand is subject to considerable volatility and inventories are replenished with non-negligible latency, as also occurs in global supply chains, the management issue becomes tedious. Moreover, in some occasions, the demand for younger/fresh products may be higher, a matter which makes the problem even more complex. Generally speaking, customers love to have fresher products that are not approaching their expiration date. In blood supply for instance, some medical conditions impose a demand that is differentiated by the age. In order to retain a high standard of quality and at the same time maintain a strict control of prices, it is important to account not only for the demand during production delay time, but also for the stock degradation in that period when placing an order. To cope with some of the above mentioned issues in perishable inventory, in this study, we address perishable products of extremely short shelf life under the assumption of differentiated demand that varies according to the age of the product. Here, old items are consumed first and only the youngest items are replenished. In case of high supply, items that are not consumed within their residual 
life will expire. Conversely, low replenishment quantity may result in shortage in any particular demanded age. The goal of this study is to decrease the scarcity and expiration of offered products by dealing with the short shelf life and the stochastic demand of all ages. An algorithm is developed that implements both Discrete Event Simulation and a stochastic search to find the optimal/near optimal order quantities at the beginning of each inventory cycle. The algorithm is expected minimize waste and avoid shortage.

This paper proceeds as follows: a literature review of the problem of perishable inventory with age discrimination is presented in section 2 followed by the methodology and the solution of the model in section 3 . The model results are demonstrated in section 4 followed by a discussion of the experiments in section 5. Finally, the conclusions are presented in section 6 .

\section{LITERATURE REVIEW}

A good deal of research exists on the problem of perishable products, which dates back to 1970s [2]. The newsvendor problem, for instance, can be considered as a typical model of short shelf life products of a single period. Research on short shelf life inventory is well established for certain problem settings, we refer the interested readers to [3] and [4] for a thorough review of this problem.

While an interesting deal of literature on stochastic demand can be found in this kind of inventory, however, age discrimination in the demand did not receive much attention, if any. In contrast, the proposed model in this paper mainly addresses age differentiation where the demand for items differ by their ages, (i.e., more demand for younger products as compared to older products). The research on the management of perishable inventory almost started in the seventies of the past century $[5,6,7$, $8,9]$. Recent surveys on retail invetory can be found in [10]. Similar surveys on perishable inventory can be found in [11].

For instance, stochastic lot-sizing problems can be found in [12] who considered perishable inventory but without age differentiation. Other earlier related research can be found in [13] and [14] who considered age distribution under base stock replenishment but no discrimination with respect to the demand. The author in [15] demonstrated a class of $(s, S)$ inventory policy to handle perishable products with no devotion to the age of products. Age differentiation is still a new area of development in the field of inventory management.

Few researchers addressed age differentiation by dividing the life time into two periods, among these researchers, we point at [16] and [17]. Different ideas can also be found in $[18,19,20,21,22]$ who considered perishable inventory management in some medical applications with little attention to age discriminated demand. In few studies, the status of inventory has been addressed using Markov chains, which occasionally were used to model perishable inventory such as [23] who used a multi-dimensional Markov chain to model the inventory circulation process.
The classical newsvendor problem as a typical model of short shelf-life inventory has been thoroughly explored in literature. Good surveys of newsvendor problem can be found in the handbook of [24] which demonstrates the adoption and the implications of the newsvendor models in miscellaneous applications and settings. The classical newsvendor problem differs from our proposal by two major suppositions, first, the newsvendor problem tackles inventory of a single period and one demand distribution, while in this proposal, multiple periods are considered along with longer expiration times. Second, the demand is discriminated by age, where younger items are often requested more than older ones, therefore, multiple distributions are used (each age has its own demand distribution).

Contrary to the presented studies above, we propose a multiple period inventory model that is characterized by age discrimination, where, different needs are realized for different ages. To be more realistic, the demand can be satisfied by products of the same age or by compatibility. Compatibility means products of other ages may still satisfy the demand of a particular age if shortage is observed. By so doing, the customers are assumed to accept older items if younger ones are not available and vice versa.

Following real applications, only the youngest items are replenished, the elderly just wait for the younger getting older. Items which are not consumed within their residual life are ultimately spoiled. Similarly, low replenishment quantity may result in shortage in any particular age. The objective of this study is to minimize the shortage and expiration in all ages by coping with the stochastic daily demand. An algorithm is developed that implements both Discrete Event Simulation and a stochastic search to find the near optimal (or probably the optimal) order quantity. The stochastic search is characterized by probabilistic acceptance/rejection of new solutions of worse moves. The search neighborhood gets narrower while navigating through the solutions until no further improvements are observed in the objective function. Different experiments were conducted to validate the model results.

\section{METHODOLOGY}

In this study, the inventory of a single product of different ages is considered under the assumption of stochastic demand. The demand may differ day-to-day, however with constant mean of each age. The products that are not sold will expire after their lifetime is consumed. Suppose that the maximum shelf life of the product is $M$ in days, hence, the product will expire within $M$ days if not already sold. Unlike some literature studies where age is differentiated by only two phases (young and old), in this paper, we will extend the shelf life to $M$, where $M$ is any positive integer. The lead-time is assumed to be 1-day, meaning that ordered quantities can be attainable a day after orders are issued. Consequently, fresh items will have an age of 1-day and only fresh items are replenished every day. Higher replenishment may yield expiration due to excessive refill rate and low consumption. On the other hand, significantly low replenishment quantities will empty the 
inventory and some ages may run short. As our model does not accept backlogs, the shortage is satisfied by expedite service of a third party. Shortage is as bad as expiration in this study with equal weights assigned to both in the objective function.

Modelling the circulation and evolvement of the inventory over time is a challenging task that has to be programmed carefully. MATLAB has been used to build the simulation model and to program the search algorithm. To test the feasibility and robustness of the proposed model and solution, a planning period of 365 days is considered with each product's age having its own demand distribution. Besides simulation, the optimization algorithm works simultaneously to find the optimal quantity that will minimize the shortage and expired amounts. In this model, it does not make difference between finding the optimal order quantity or finding the optimal orderup-to-level as both are the same for one reason, this is, the inventory age 1 (i.e., fresh items) will be age 2 next day, hence, the inventory of age 1 will always be " 0 " when the time for replenishment comes the next day, as shown in Fig. 1. We will use the two terms interchangeably. Anyway, the task of the decision maker is to determine the optimal daily replenishment quantity of the youngest items to keep shortage and expiration at minimum levels.

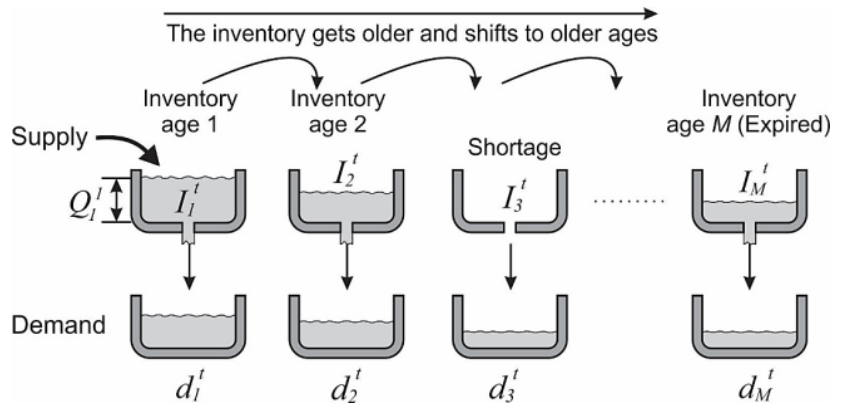

Fig. 1 The inventory status and demand in a typical day $t$

In typical day $t$, the inventory of all ages is given by $I_{1}^{t}, \ldots, I_{M}^{t}$, and the total inventory of the same product of all ages is $\sum_{i=1}^{M} I_{i}^{t}$. Orders for the different ages is satisfied as follows: first, the demand is satisfied by the exact match starting from the youngest (i.e., items of the same age), if the exact match is not enough, a substitute of other ages is found (i.e., age mismatch). Likewise, the demand for older items can be satisfied by youngers items if no exact age match is found. The demand that is satisfied by age mismatch follows older-and-closer-in-age policy with the priority in get substitutes belonging to the younger items. This assumption is important as it guarantees the consumption of older items first. The order fulfillment policy is described in Table 1. The columns show the preferred ages to be used in satisfying the demand ( 1 being the most preferred in each column).

For better demonstration, consider a maximum shelf life of $M=6$ days, the resulting preference for this instance is given in Table 2. Note, the diagonal of the table shows the most preferred ages. For example, product age 1 prefers age 1 as a first alternative, the same applies for the other ages. The next preferred alternative is the one that is closer in age but older. For example, if we consider the demand of the recipient age 4 , products of age 4 are preferred first, then age 5 followed by 6 , then the ages 3,2 , 1 , respectively. When two recipients' ages compete for one substitute (a situation that may occur when two recipients run short), younger items get the substitute first.

Table 1

Demand preference across the supply, (1 is most preferred) Recipient Demand

\begin{tabular}{|c|c|c|c|c|c|c|c|c|}
\hline \multirow{8}{*}{$\begin{array}{l}\text { 추 } \\
\frac{0}{3} \\
\text { ज } \\
\frac{1}{0} \\
\frac{1}{0} \\
0\end{array}$} & age & 1 & 2 & 3 & $\ldots$ & M-2 & M-1 & $M$ \\
\hline & 1 & 1 & $M$ & $M$ & $\ldots$ & $M$ & $M$ & $M$ \\
\hline & 2 & 2 & 1 & $M-1$ & $\ldots$ & $M-1$ & $M-1$ & $M-1$ \\
\hline & 3 & 3 & 2 & 1 & & $M-2$ & $M-2$ & $M-2$ \\
\hline & $\vdots$ & $\vdots$ & $\vdots$ & $\vdots$ & $\because$ & $\vdots$ & $\vdots$ & $\vdots$ \\
\hline & M-2 & $M-2$ & $M-3$ & $M-4$ & & 1 & 3 & 3 \\
\hline & M-1 & $M-1$ & $M-2$ & $M-3$ & $\ldots$ & 2 & 1 & 2 \\
\hline & $\mathbf{M}$ & $M$ & $M-1$ & $M-2$ & $\ldots$ & 3 & 2 & 1 \\
\hline
\end{tabular}

Table 2

An instance of preference for a maximum shelf life of 6 days Recipient

\begin{tabular}{llllllll} 
& \multicolumn{7}{c}{ age } \\
\hline & age & $\mathbf{1}$ & $\mathbf{2}$ & $\mathbf{3}$ & $\mathbf{4}$ & $\mathbf{5}$ & $\mathbf{6}$ \\
& $\mathbf{1}$ & 1 & 6 & 6 & 6 & 6 & 6 \\
& $\mathbf{2}$ & 2 & 1 & 5 & 5 & 5 & 5 \\
$\overline{0}$ & $\mathbf{3}$ & 3 & 2 & 1 & 4 & 4 & 4 \\
$\bar{\circ}$ & $\mathbf{4}$ & 4 & 3 & 2 & 1 & 3 & 3 \\
& $\mathbf{5}$ & 5 & 4 & 3 & 2 & 1 & 2 \\
& $\mathbf{6}$ & 6 & 5 & 4 & 3 & 2 & 1 \\
\hline
\end{tabular}

A superior inventory policy is a one that maintains mismatch at " 0 " levels. However, as the demand is stochastic and the product gets older, it will be almost impossible to avoid age mismatch. In fact, if no mismatch compatibility policy is employed, higher shortage and expiration rates will be observed.

A single product that is characterized by different ages, $a=1, \ldots, M$, is considered. Every day $t$, a random demand is realized for each age which is given by the vector $D^{t}=\left[d_{1}^{t}, \ldots, d_{M}^{t}\right]$. The demand follows Poisson distribution of means that differ by age, that is, each age has one

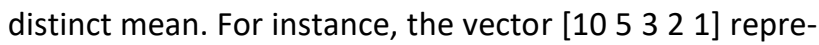
sents the means of the ages 1 through 5 , respectively. The mean demand for younger ages is usually higher than older ages. Beginning of day $t$, the status of the inventory can be described by the following vector:

where:

$$
I^{t}=\left[\begin{array}{c}
I_{1}^{t} \\
\vdots \\
I_{M}^{t}
\end{array}\right]
$$

$I_{a}^{t}$ stands of the available inventory of age " $a$ " at day $t$. After the realization of the demand, orders are satisfied by exact age match first, as follows:

$$
I_{a}^{t}=\left[I_{a}^{t}-d_{a}^{t}\right]^{+}, \quad \forall a=1, \ldots, M
$$

where:

$[z]^{+}$refers to $\max (0, z)$. 
If the available quantities are not enough for exact age match, substitutes of other age are found according to Table 1 and following the rules below:

- Younger items have higher priority in getting substitutes.

- Substitutes are selected according to older-andcloser-in-age policy. This will reduce the difference in age between the donors and recipients, thereby, trying to trade same product generations.

- Items of age $M$ are spoiled right after satisfying the demand by exact as well as by age mismatch.

- Only young items are replenished, the other ages get their supply from leftovers as younger items get older.

- Items get older day-to-day, until they are consumed or ultimately spoiled due to expiration.

Age mismatch will alter the inventory status as follows (i.e., after performing equation 1 ):

$$
I_{a}^{t}=\left[I_{a}^{t}-C I^{t}(a)\right]^{+}, \forall a=1, \ldots, M,
$$

where:

$C I^{t}(a)$ is the consumed inventory of age " $a$ " to satisfy the demand of other ages.

Two main steps are left leading to the conclusion of shortage and spoiled quantities of each age as illustrated below:

Step 1: Product shortage of all ages:

$$
S_{a}^{t}=\left[\left[d_{a}^{t}-I_{a}^{t}\right]^{+}-S I^{t}(a)\right]^{+} \quad \forall a=1,2, \ldots, M
$$

where:

$S I^{t}(a)$ is the supplied inventory to other ages that is used in satisfying the demand of age $a$.

Step 2: Expired amounts:

Upon satisfying the demand by both exact and mismatch operations, the expired amounts are those of age $M$ which are not consumed end of $t$, this is:

$$
E_{M}^{t}=I_{M}^{t}
$$

The replenishment quantity is the amount that brings the inventory of the youngest items back to $Q_{1}^{t}$. For further demonstration, consider the example presented in Table 3. Assume that the order up to level $Q_{1}^{t}=5$ items. Of note, the order-up-to-level can be denoted by $Q_{a}^{t}$ if the other ages are replenished, however, only those of age 1 are replenished in this study.

Table 3

An example: Perishable inventory of a lifetime of 4 days

\begin{tabular}{lccc}
\hline \multicolumn{1}{c}{$\begin{array}{c}\text { Initial inventory: } \\
(\mathbf{4 , 2 , 4 , 1 )}\end{array}$} & $\begin{array}{c}\text { Day } \mathbf{1} \\
\text { Demand: } \\
(\mathbf{3 , 1 , 2 , 2 )}\end{array}$ & $\begin{array}{c}\text { Day } \mathbf{2} \\
\text { Demand: } \\
(\mathbf{3 , 0 , 1 , 0 )}\end{array}$ & $\begin{array}{c}\text { Day } \mathbf{3} \\
\text { Demand: } \\
(\mathbf{2}, \mathbf{3 , 4 , 0})\end{array}$ \\
\hline $\begin{array}{l}\text { Inventory after exact } \\
\text { match }\end{array}$ & $1,1,2,0$ & $2,1,0,1$ & $3,0,0,0$ \\
$\begin{array}{l}\text { Inventory after } \\
\text { compatibility match }\end{array}$ & $1,1,1,0$ & $2,1,0,1$ & $0,0,0,0$ \\
$\begin{array}{l}\text { Shortage end } \\
\text { of period } t\end{array}$ & $0,0,0,0$ & $0,0,0,0$ & $0,0,1,0$ \\
$\begin{array}{l}\text { Expired end of period } t \\
\text { Replenishment } \\
\text { of youngest age } Q_{1}^{t}\end{array}$ &,,,--- 0 &,,,--- 1 &,,,--- 0 \\
$\begin{array}{l}\text { Inventory ready } \\
\text { for next day }\end{array}$ & 5 & 5 & 5 \\
\hline
\end{tabular}

The initial inventory of the above example is given by $(4,2,4,1)$ beginning of day 1 . Clearly, the demand for all ages can be satisfied by exact age match except for age 4 , where a quantity of 1 remains short which is satisfied by compatibility from items of age 3 . The remaining inventory end of day 1 is $(1,1,1,0)$. Since the demand has been completely satisfied by both exact and age mismatch, no shortage is observed. Similarly, the leftover (age 4) is also " 0 ". The remaining will get older by one day and a quantity of 5 items of age 1 arrives. This is updated as $(1,1,1,0)$ $\rightarrow(0,1,1,1) \rightarrow(5,1,1,1)$ which will be available for day 2 . The demand in day 2 can be satisfied by exact match without the need for substitutes. However, one item of age 4 will expire and 5 new items will be replenished. Therefore, the available inventory for day 3 will be $(5,2,1,0)$. Day 3 will have a shortage in those age 3 even with mismatch substitution. The same inventory policy repeats every day. Clearly, the size of the daily replenishment affects both shortage and expiration rates.

A consumption matrix $C^{t}$ can be construct for every transition period as follows:

$$
C^{t}=\overbrace{\left[\begin{array}{ccc}
c_{1,1}^{t} & \cdots & c_{1, M}^{t} \\
& \ddots & \\
c_{M, 1}^{t} & \cdots & c_{M, M}^{t}
\end{array}\right]}^{\text {age }=1}
$$

The matrix shows the amounts consumed by exact match (the diagonal) and age mismatch (upper and lower elements). Each column in the matrix represents the amounts used to satisfy its demand, while each row represents the amount consumed from the row's corresponding age to satisfy the others. For instance, items age $M$ are satisfied by the exact match quantity $\left(c_{M M}^{t}\right)$ in addition to the quantities attained from other ages: $c_{1 M}^{t}$ through $c_{M-1, M}^{t}$.

The above matrix is updated on a daily basis with each day having its own consumption matrix. The grand sum over the planning period $T$ is given by:

$$
\boldsymbol{C}=\sum_{t=1}^{T} C^{t}=\left[\begin{array}{ccc}
\boldsymbol{c}_{11}^{t} & \cdots & \boldsymbol{c}_{1 M}^{t} \\
& \ddots & \\
\boldsymbol{c}_{M 1}^{t} & \cdots & \boldsymbol{c}_{M M}^{t}
\end{array}\right]
$$

The matrix $\boldsymbol{C}$ gives the total amounts used to satisfy the demand detailed to each age. It also gives the supply distribution (columns) and the consumption distribution (rows). For instance, the first column shows the amounts used to satisfy the demand of age 1 in the entire planning period $T$. The quantities used as exact match are given by $\sum_{a=1}^{M} \boldsymbol{C}_{a a}$, while those consumed in covering the demand by younger items equal to $\sum_{a=1}^{M} \sum_{b=1}^{M} \boldsymbol{C}_{a a}, \forall a>b$. Likewise, those consumed to meet the demand by older items are given by $\sum_{a=1}^{M} \sum_{b=1}^{M} \boldsymbol{C}_{a a}, \forall a<b$. One performance measure that should be addressed is the mismatch ratio. Keeping the mismatch ratio at minimum levels means higher satisfaction by exact ages. The total mismatch ratio is given by:

$$
\text { Mismatch ratio }=\frac{\sum_{a=1}^{M} \sum_{b=1}^{M} \boldsymbol{C}_{a b}-\sum_{a=1}^{M} \boldsymbol{C}_{a a}}{\sum_{a=1}^{M} \sum_{b=1}^{M} \boldsymbol{C}_{a b}}
$$

While different cost objective functions can be modeled to optimize the inventory, however, shortage and expiration are the key performance measures. Minimization of 
shortage and expiration over the entire planning period is our objective function of choice. In this study, equal weights are assigned for both measures, this is:

$$
\operatorname{Min} \sum_{t=1}^{T}\left(\sum_{a=1}^{M} S_{a}^{t}+E_{M}^{t}\right)
$$

The objective function presented in (8) cannot be evaluated analytically as it depends on the simulation results of each run of the entire planning period. Therefore, while the optimization algorithm searches for the best values, the simulation model will be tested for multiple times to get an estimate of the shortage and expiration. The concurrent values of shortage and expiration will determine the right search directions accordingly. Simulation is also important to generate the demand stream out of Poisson distribution; therefore, beginning of each simulation run, a data vector is generated for the realization of the demand day by day.

The circulation of inventory and the optimization algorithm have been coded using MATLAB. Random numbers library is used to get the random demand and to operate the stochastic search algorithm. The solution space is the feasible range which is restricted to non-negative values and sufficiently high upper bounds. The best order-up-tolevel quantities are those which will minimize both shortage and expiration equally likely. For every new solution, the model has to be simulated once to get the resulting shortages and expired amounts for guided search paths. Along with the evaluation of the objective function, the mismatch matrix is evaluated as another performance measure of the model.

While the optimization algorithm navigates through the different solutions, each is evaluated via simulation as shown in Fig. 2. For every iteration in the optimization side, the inventory is simulated for a planning period of $T$.

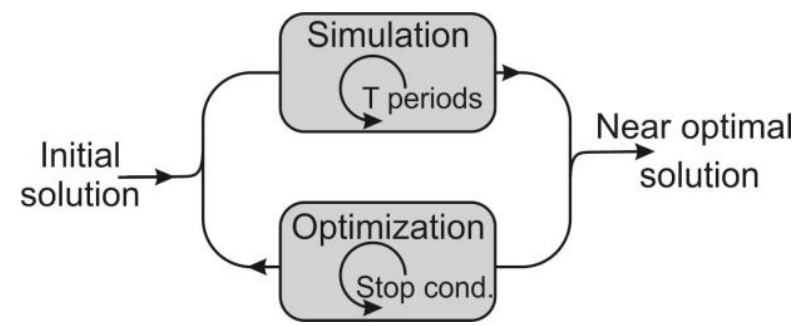

Fig. 2 A schematic diagram of simulated optimization

Due to stochastic settings in the model, challenges may arise in finding the best solution neighborhood, a matter that requires tuning the search parameters in few initial trials for faster convergence. Although the search algorithm is stochastic in accepting better/worse solutions, the search directions are sufficiently limited to make a simple stochastic exploration efficient in finding the optimal values. Exclusive tracking of inclined gradients of the objective function does not guarantee hill combing, therefore, to avoid the trap in potential minima, worse solutions can be probabilistically accepted. Upon the acceptance of a new move, the solution is evaluated once for a period of $T$. Fig. 3 demonstrates a simple pseudo code of the algorithm.

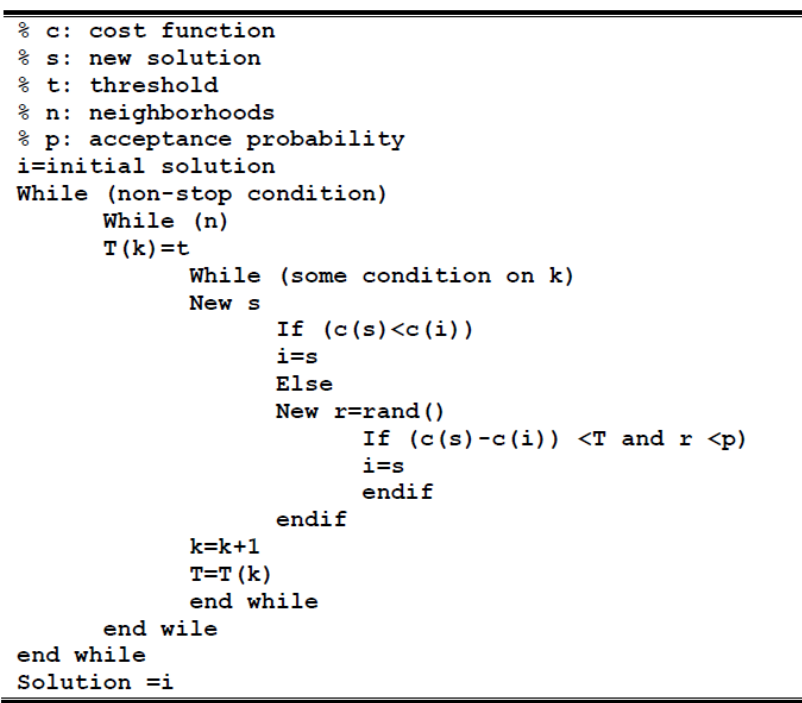

Fig. 3 Pseudo code of the optimization algorithm

\section{RESULTS}

To verify and validate the proposed model and solution algorithm, a short shelf life inventory of 1-day lifetime is considered. Of note, when a maximum shelf life of 1-day is considered (i.e., the inventory has to be consumed the same day it is ordered, otherwise spoiled), the problem reduces to a classical newsvendor. We have tested our model for 1-day lifetime and compared our results with that attained by the classical newsvendor given normal demand $N(\mu, \sigma)$ and similar overage and underage costs. Our results match with those found in the analytical newsvendor to an extent of almost $99 \%$ in 30 trials each simulated for 365 days. The Mean Absolute Percent Error (MAPE) is $\approx 1 \%$. Table 4 shows the analytical and simulated optimization results (i.e., newsvendor problem). The optimal quantities are remarkably close to those found using the analytical solution. (The reader is referred to the classical newsvendor for details).

Table 4

Analytical solution of the newsvendor vs. simulated optimization. (Normal demand and similar overage and under costs re used in the newsvendor problem. Similar shortage and expiration weights are used in the simulated optimization)

\begin{tabular}{ccccc}
\hline $\begin{array}{c}\text { Mean } \\
(\boldsymbol{\mu})\end{array}$ & $\begin{array}{c}\text { Standard } \\
\text { deviation } \\
(\boldsymbol{\sigma})\end{array}$ & $\begin{array}{c}\text { Newsvendor } \\
\text { optimal } \\
\text { quantity }\end{array}$ & $\begin{array}{c}\text { Simulated } \\
\text { optimiza- } \\
\text { tion }\end{array}$ & MAPE \\
\hline 10 & 2 & 10 & 9.85 & 0.015 \\
20 & 4 & 20 & 20.2 & 0.01 \\
30 & 6 & 30 & 29.67 & 0.011 \\
40 & 8 & 40 & 40.56 & 0.014 \\
50 & 10 & 50 & 51.01 & 0.0202 \\
10 & 4 & 10 & 10.1 & 0.01 \\
20 & 6 & 20 & 19.85 & 0.0075 \\
30 & 8 & 30 & 29.52 & 0.016 \\
40 & 10 & 40 & 40.7 & 0.0175 \\
50 & 12 & 50 & 49.31 & 0.0138 \\
& & & Average & $1.35 \%$ \\
\hline
\end{tabular}

Having the model validated by a standard analytical solution, next, we proceed to consider products of $2,3,4$, and 5 days lifetime. A planning period of 365 days is selected 
for each lifetime experiment. Each experiment is simulated 30 times (i.e., a total of 30 years of simulation). Thirty trials are enough to test our confidence in the results for different demand streams. Narrower confidence intervals mean that the solutions are consistent and fall within same optimal neighborhood. On the other hand, if the confidence interval is relatively wide, the chances that some solution may have been trapped in a local minimum are higher. In our experiments, narrow confidence intervals were observed as detailed down this section.

For benchmark comparison, in the remaining four experiments we assume that the sum of the demand means for all ages is the same, that is, $D_{\max }=10$ items/day. The distribution of $D_{\max }$ over the different ages is decreasing so as to hold the proposition that older items are not as attractive as new ones.

First experiment: A single product of a short shelf life of 2 days is considered, hence, the product has to be consumed within 2 days since replenishment, otherwise, will be spoiled. In this experiment, the demand follows Poisson distribution of the means $(6,4)$ items/day for both ages, and only young units are replenished. Of note, this inventory problem cannot be analytically solved as in the case of classical newsvendor as there are two periods and two demand distributions of different means. Both age 1 and age 2 are compatible as demonstrated in Table 1.

Second experiment: An extension of the above scenario is addressed here with a shelf life of 3 days. Only items of age 1 are replenished, while items of age 3 days that remain in inventory are spoiled. Poisson distribution is used for the demand with the following means $(5,3,2)$ items/day. Substitution by compatibility follows the guidelines presented in Table 1.

Third experiment: The second experiment is extended to a shelf life of 4 days. Only items of age 1 are replenished, while items of age 4 remaining in inventory are spoiled. Substitution by compatibility follows the guidelines stated in Table 1 . With a sum of $D_{\max }=10$, the demand means for this case are $(4,3,2,1)$ items/day.

Fourth experiment: Although our model can be extended to any lifetime, however, the interest of this research is to tackle the inventory of extremely short lifetimes (up to 5 days). A particular attention will be paid to this case as it can be applied to Platelets Apheresis inventory in Oncology and Hematology field. Dairy products and ready food are other typical examples that exhibit such a short lifetime. Anyway, blood platelets can survive for only 5 days end of which they are either consumed or spoiled. Platelets are precious products that must be managed with care as shortage and expiration are so destructive. For this experiment, the following means were selected: $(4,2,2,1,1)$ items/day. Similar mean distributions can be found in literature. In blood organizations, it is usually preferred to get the exact platelet age match of each order, however, as the demand is stochastic, it is highly unlikely to satisfy all orders by exact match, therefore, the platelets that are older but closer-in-age are used (as in Table 1). Certainly, in such medical application, the impact of spoiling such living cells is detrimental. On the other hand, shortage may lead to loss of lives as well. Accordingly, both shortage and expiration have significant adverse effects on the performance of the inventory. We would like to note that; cost models for such precious living tissue are not so suitable, thereby, adhering to human trafficking international laws.

\section{DISCUSSION OF THE FOUR EXPERIMENTS}

Our results are based on 30 experiments for each lifetime, each of which is simulated for 365 days. The optimal objective function (shortage and expiration) is recorded for each lifetime as shown in Fig. 4.

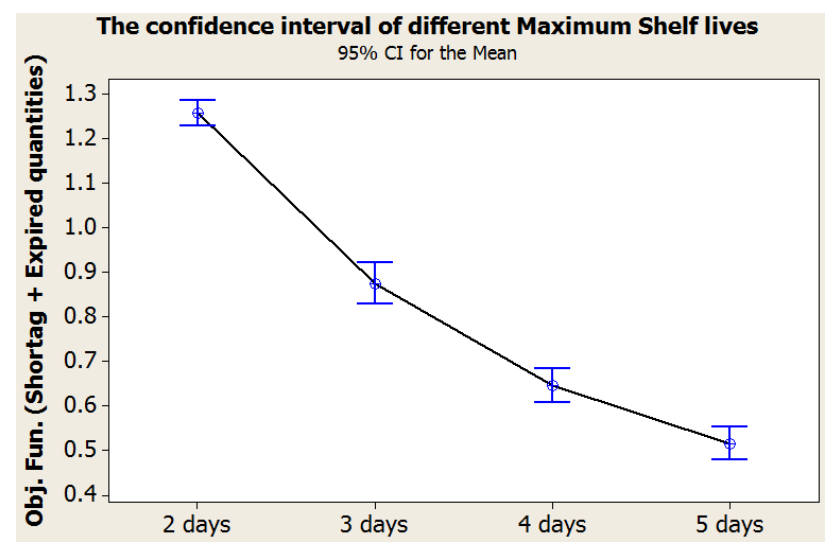

Fig. 4 The objective function value and the $\mathrm{Cl}$ for different lifetimes

Evidently, shorter lifetimes yield worse performance as there is not long time till consumption, hence the products have higher chances of being spoiled. Longer shelf lives, on the other hand, provide more tolerance and thereby, longer time for consumption. Fig. 5 shows the objective function value and its confidence interval scaled down to shortage and expiration/day. Noticeably, the objective function value significantly decreases to low levels of shortage and expiration as the maximum shelf life increases. The confidence intervals are remarkably tight around the averages with no outliers, meaning that no local minimum is trapping an outlier solution, Table 5.

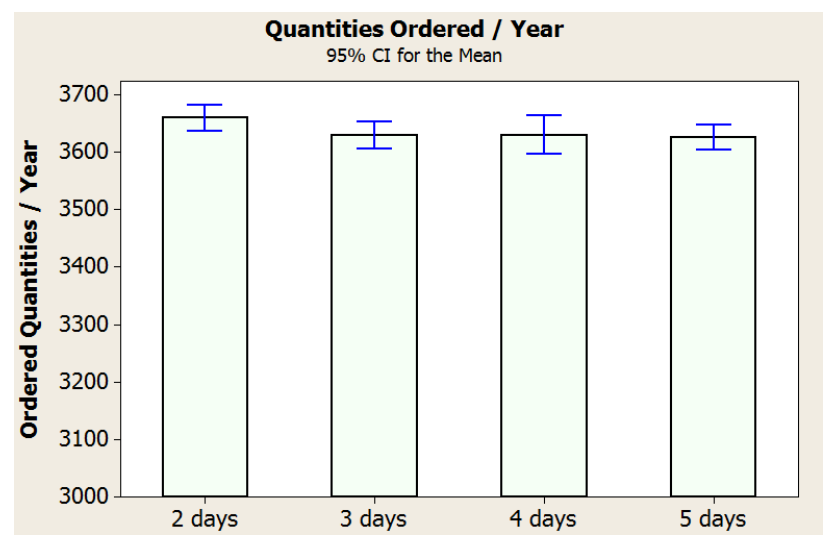

Fig. 5 The confidence interval of the ordered quantities for different maximum shelf lives

Only 0.5 items will outdate or will go short in a shelf life of 5 days which constitutes $5 \%$ of the demand. 
Table 5

The resulting objective function (shortage + expired) for the 4 experiments

\begin{tabular}{ccccc}
\hline Shelf Life & Mean & St. dev. & \multicolumn{2}{c}{ Confidence Interval (95\%) } \\
\hline 2 & 3659.7 & 60.6 & 3637.1 & 3682.4 \\
3 & 3629.3 & 61.9 & 3606.2 & 3652.4 \\
4 & 3629.3 & 89.2 & 3596.0 & 3662.6 \\
5 & 3625.7 & 58.6 & 3603.8 & 3647.5 \\
\hline
\end{tabular}

The annual ordered quantities are shown in Fig. 5. Slight fluctuations around 3650 are observed as the sum of all Poisson averages is 10 items/day. Note the quantities ordered in the case of 2 days lifetime is little higher due to higher chances of expiration.

The average of the optimal decision variable is shown in Fig. 6 which demonstrates tight confidence intervals for the 30 trials and an average of almost 10 items/day.

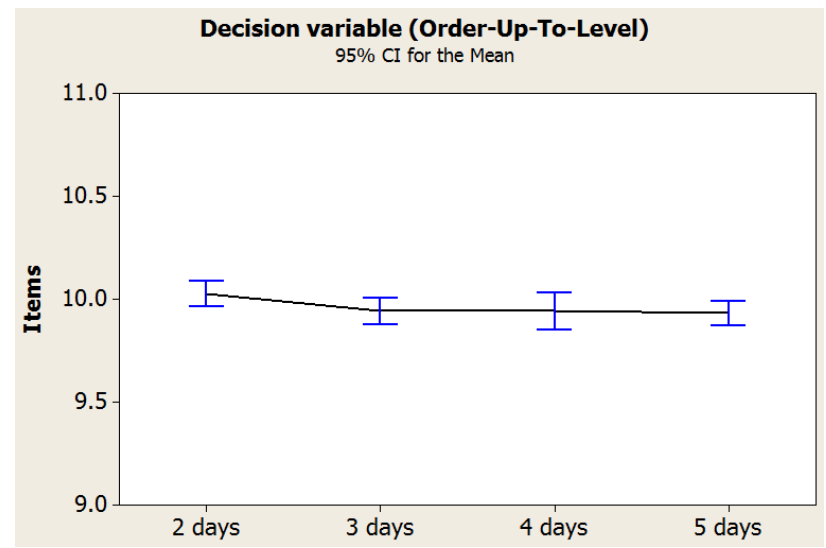

Fig. 6 Near optimal replenishment quantities/day for the 4 experiments

The replenishment quantities resulting in this figure demonstrate the robustness of the search algorithm and its ability to drive the inventory to optimality neighborhood of minimum levels of shortage an expiration under stochastic demand.

One more experiment is conducted for a shelf life of 5 days. Shortage and expiration detailed to each shelf life are given in Fig. 7. Both shortage and expiration decrease by increasing the maximum shelf life exhibiting the same pattern. Note that shortage will be less than $1 \%$ of the demand with almost similar ratio for the expiration.

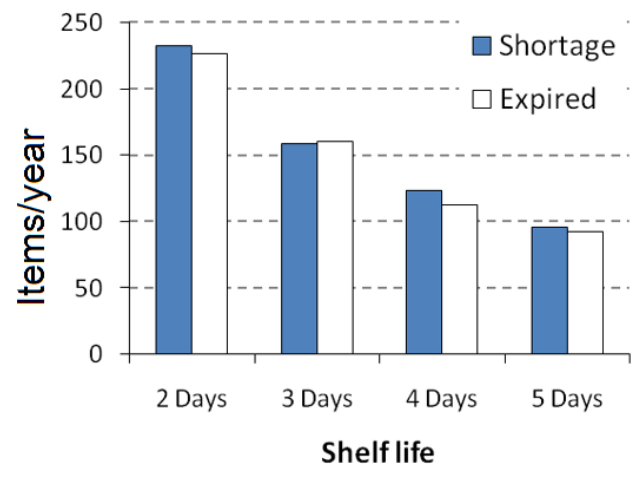

Fig. 7 The objective function detailed to shortage and expiration
Fig. 8 shows the percent of satisfied demand by exact age for all scenarios. For example, the bars belonging to age $=$ 1 show the percent of items satisfied by exact match for the scenarios of 2, 3, 4 and 5 lifetime periods. Particularly, the first bar in the graph belongs to products of a shelf life of 2 days. It informs that almost $80 \%$ of items aged 1-day are satisfied by exact age match. Similarly, for the category of 5 days shelf life, the demand for those age 5 of this type is $75 \%$ satisfied by exact match as shown by the last bar in the figure.

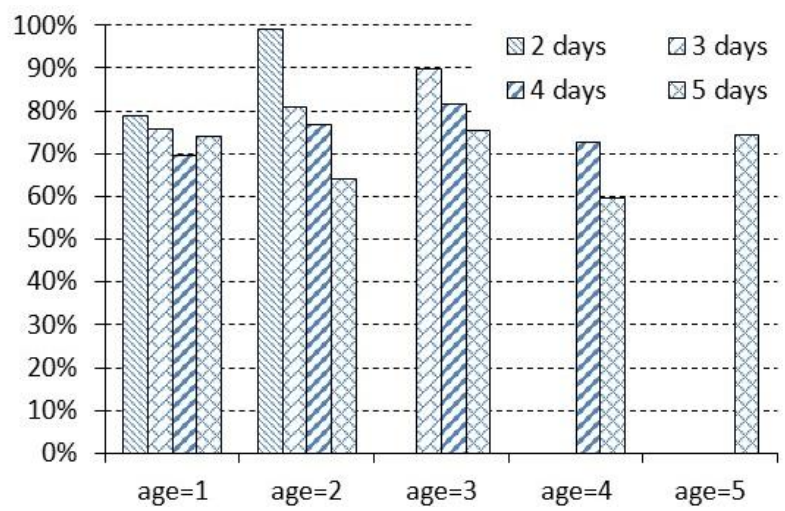

Fig. 8 Percent of demand satisfied by exact age match for all shelf lives

The supply-demand point of views are shown in Fig. 9 and Fig. 10.

Fig. 9 presents the supply for each age, for example, age 1 is $74 \%$ supplied by exact match and the remaining by compatibility. Note that as the lifetime increases, more chances for compatible operations can be observed.

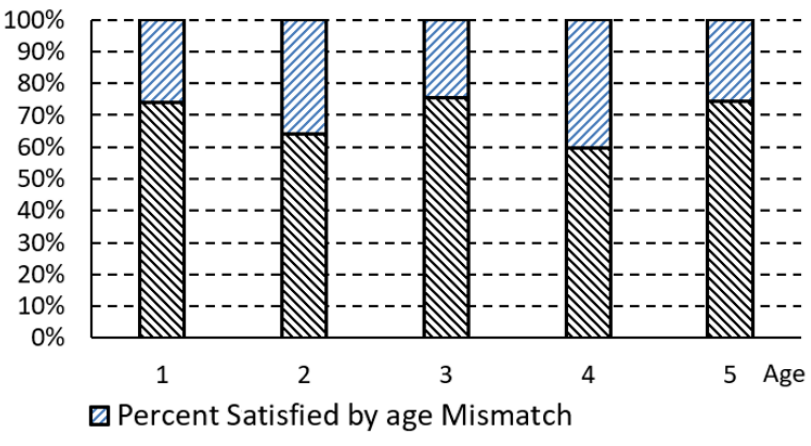

ه Percent Satisfied by Exact Match

Fig. 9 Supply by exact and age mismatch of each age type

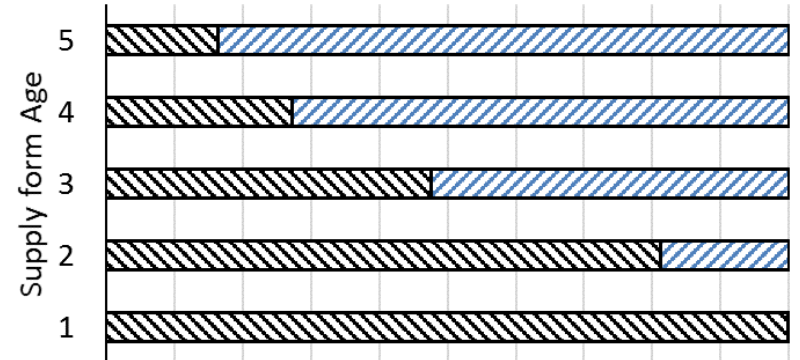

$\begin{array}{lllllllllll}0 \% & 10 \% & 20 \% & 30 \% & 40 \% & 50 \% & 60 \% & 70 \% & 80 \% & 90 \% & 100 \%\end{array}$

\$Self Supply $\square$ Supply to other ages

Fig. 10 Supply distribution of each age 
Similarly, by consider the consumption distribution in Fig. 10 , we realize that older items mostly serve as donors while younger items present more greed. For example, $18 \%$ of those age 5 are consumed by the demand of this age and a remaining of $82 \%$ is used to satisfy the other ages. While Fig. 9 shows that out of all quantities satisfying age 1 , only $74 \%$ of this age is satisfied by its own kind, however, Fig. 10 shows also that almost $100 \%$ of those age 1 are consumed by the same age, meaning that products age 1 are not generous donors and those age 5 are the best donors.

\section{CONCLUSIONS}

This paper builds upon the newsvendor problem where a multi-period perishable inventory of extremely short shelf life is considered. In contrast to majority of existing literature, here, the demand is stochastic and discriminated by age, thereby, newer items exhibit higher average demand as compared to older ones. The product gets older day by day till consumption or expiration. The demands are satisfied by exact age match first, then by compatibility if exact age match is not available. The inventory policy follows certain guidelines to mimic real situations such as RBC's inventory in blood banks. The goal is to find an optimal replenishment quantity such that shortages and expirations are minimized.

The supplier replenishes only younger items while old ones get their quantities by aging of younger ones. Higher replenishment quantities yield expiration, while low quantities my result in shortage. The model objective function is to minimize both quantities via simulated optimization approach. Discrete Event Simulation model is built to model the inventory circulation over a planning period of 1 year. Five different shelf lives were considered, starting from the classical newsvendor up to products of 5 days lifetime, each has been simulated for 30 times. The search algorithm employs stochastic moves while navigating in the solution space. The stochastic search allows for hill climbing and avoiding local optimal solutions.

The simulation experiment revealed remarkable results of great consistency. It was found that both shortage and expiration decrease by increasing the shelf life (down to $1 \%$ of the demand for a shelf life of 5 days). Mismatch substitutions also decrease by increasing the maximum shelf life. The confidence intervals are found to be remarkably narrow, a matter that assures consistency and inexistence of outlier solutions, thereby, reassuring optimality neighborhood. Slight fluctuations around the average demand were observed in optimal quantity indicating a good deal of consistency in the solution.

Finally, while the proposed model identifies the products by different ages, one limitation that may exist is the unavailability of demand data for each age. Moreover, higher fluctuations in the demand of each age may result in inflated bullwhip effect which can be addressed in future extension of this work. Another limitation, the proposed model works well for products of extremely short shelf lives. If the products exhibit longer shelf lives, the products may no longer be discriminated by age and hence another model may be more suitable for this category of problems.

\section{REFERENCES}

[1] L. McNeill. (2011). "The retail market: Challenges \& opportunities" IBM, Hampshire, UK. Available: http://www03.ibm.com/systems/data/flash/retail/resources/Inventory_Risk_Paper.pdf.v.

[2] S. Nahmias. "Perishable inventory theory: a review" Operations Research, vol. 30, 1982, pp. 680-708.

[3] I.Z. Karaesmen, A. Scheller-Wolf, B. Deniz. "Planning Production and Inventories in the Extended Enterprise". International Series in Operations Research \& Management Science, vol. 151, 2011, pp. 393-436.

[4] X. Chao, X. Gong, C. Shi, H. Zhang. "Approximation algorithms for perishable inventory systems". Operations Research, vol. 63, no 3, 2015, pp. 585-601.

[5] S.D. Pinson, W.P. Pierskalla, B. Schaefer "A computer simulation analysis of blood bank inventory policies". Technical Report, Department of Industrial Engineering and Management Sciences, Northwestern University, Evanston, Illinois, 1972.

[6] J.B, Jennings, "Blood bank inventory control". Management Science, vol 19, 1973, pp. 637-645.

[7] M.A. Cohen, W.P. Pierskalla. "Management policies for a regional blood bank". Transfusion, vol 15, 1975, pp. 58-69.

[8] C.P. Schmidt, S. Nahmias. "(S-1, S) policies for perishable inventory". Management Science, vol 31, 1985, pp. 719728.

[9] D. Perry, M.J.M Posne. "An (S-1, S) inventory system with fixed shelf life and constant lead-times". Operations Research, vol 46, 1998, pp. 565-571.

[10] A. Johnston. "Trends in retail inventory performance: 1982-2012". Operations Management Research, Volume 7, Issue 3-4, 2014, pp. 86-98.

[11] S. Nahmias. "Perishable Inventory Systems" Springer International Series in Operations Research \& Management Science, vol. 160, 2011.

[12] R. Levi, C. Shi. "Approximation algorithms for the stochastic lot-sizing problem with order lead times". Operations Research, vol. 61, no 3, 2013, pp. 593-602.

[13] D. Chazan, S. Gal. "A Markovian model for a perishable product inventory". Management Science, vol 23, 1977, pp. 512-521.

[14] M.A. Cohen. "Analysis of single critical number ordering policies for perishable inventories". Operations Research, vol 24, 1976, pp. 726-741.

[15] Z. Lian, L. Liu, M.F. Neuts. "A discrete-time model for common lifetime inventory systems". Mathematics of Operations Research, vol 30, no 3, 2005, pp. 718-732.

[16] R. Haijema, J. van der Wal, \& N. van Dijk. "Blood platelet production: Optimization by dynamic programming and simulation". Computers and Operations Research, vol 34, no 3, 2007, pp. 760-779.

[17] H. Zhang, C. Shi, X. Chao. "Technical Note - Approximation Algorithms for Perishable Inventory Systems with Setup Costs". Informs operations research, 2016, pp. 432-440.

[18] Q. Duan, T.W. Liao. "A new age-based replenishment policy for supply chain inventory optimization of highly perishable products". International Journal of Production Economics, vol 145, 2014, pp. 658-671. 
[19] S.A. Glynn. "The red blood cell storage lesion: A method to the madness". Transfusion, vol 50, no 6, 2010, pp. 11641169.

[20] D. Zhou, Leung, L.C., W.P. Pierskalla. "Inventory management of platelets in hospitals: Optimal inventory policy for perishable products with regular and optional expedited replenishments". Manufacturing and Service Operations Management, vol 13, no 4, 2011, pp. 420-438.

[21] A. Gutierrez-Alcoba, G. Ortega, M.T. Eligius, I. García. “Accelerating an algorithm for perishable inventory control on heterogeneous platforms Original Research Article". Journal of Parallel and Distributed Computing, vol 104, 2017, pp. 12-18.

\section{Wasfi Alrawabdeh}

ORCID ID: 0000-0002-1172-7622

The Hashemite University Department of Management

Faculty of Economics and Administrative Sciences P.O. Box 150459, Zarqa 13115, Jordan

e-mail: rawabdeh@hu.edu.io
[22] D. Dalalah, O. Bataineh, K. Alkhaledi. "Platelets inventory management: A rolling horizon Sim-Opt approach for an age-differentiated demand". Journal of Simulation, vol 13, Issue 3, 2019, pp. 209-225.

[23] Z. Lian, L. Liu. "A discrete-time model for perishable inventory systems". Annual of Operations Research, vol 87, 1999, pp.103-116.

[24] T.M. Choi. "Handbook of Newsvendor Problems: Models, Extensions and Applications". Springer Science \& Business Media, 2012. 